\title{
Conventional ultrasonography and sonoelastography in the assessment of plaque psoriasis under topical corticosteroid treatment - work in progress.
}

\author{
Maria Cucoş $^{1}$, Maria Crişan², Manuela Lenghel ${ }^{1}$, Marina Dudea ${ }^{1}$, Roxana Croitoru ${ }^{2}$, Sorin \\ M. Dudea ${ }^{1}$
}

${ }^{1}$ Radiology Department, ${ }^{2}$ Histology Department, "Iuliu Haţieganu” University of Medicine and Pharmacy, ClujNapoca, Romania

\begin{abstract}
Aims: The objective of the study was to compare the efficiency of $20 \mathrm{MHz}$ skin ultrasonography and $40 \mathrm{MHz}$ conventional ultrasonography in the assessment of plaque psoriasis and to assess the efficiency of $40 \mathrm{MHz}$ real-time sonoelastography in the early detection of steroid-induced skin atrophy in psoriatic plaques. Patients and methods: Ultrasonographic blinded evaluation was performed on 16 plaques in three consecutive patients, at baseline and after hydrocortisone acetate $1 \%$ ointment six-week application. The parameters were epidermal and dermal thicknesses for gray-scale ultrasonography and strain ratio for sonoelastography. Strain ratio was computed between the dermis and the adjacent hypodermis. Student's t-test for paired samples was performed. A confidence level of $\mathrm{p}<.05$ was considered significant. Results: At follow-up, epidermal thickness was significantly reduced with $20 \mathrm{MHz}(\mathrm{p}=.002)$ and $40 \mathrm{MHz}$ sonography $(\mathrm{p}=.032)$, while dermal thickness varied insignifcantly with $20 \mathrm{MHz}(\mathrm{p}=.35)$ and $40 \mathrm{MHz}$ sonography $(\mathrm{p}=.33)$. Measurements at $40 \mathrm{MHz}$ were significantly higher than their $20 \mathrm{MHz}$ counterparts at baseline (epidermis: $\mathrm{p}<.001$; dermis: $\mathrm{p}=.003$ ) and at follow-up (epidermis: $\mathrm{p}<.001 ;$ dermis: $\mathrm{p}$ $=.001)$. Strain ratio revealed no significant change of dermal elasticity $(\mathrm{p}=.96)$. Conclusions: Although epidermal and dermal measurements varied significantly with $20 \mathrm{MHz}$ and $40 \mathrm{MHz}$ ultrasonography, both techniques efficiently quantified treatment response by measuring the reduction of epidermal thickness. Sonoelastographic findings were consistent with the expected outcome of short-term use of low-potency corticosteroid. Further studies are recommended.
\end{abstract}

Keywords: psoriasis, ultrasonography, sonoelastography, corticosteroid, skin atrophy

\section{Introduction}

Psoriasis is a chronic, non-infectious, inflammatory disease of the skin, characterized by well-defined erythematous plaques, bearing adherent, silvery scales [1,2]. The plaque pattern is the most common type. The sites of

Received 12.03.2014 Accepted 9.04.2014

Med Ultrason

2014, Vol. 16, No 2, 107-113

Corresponding author: Maria Crişan

Histology Department

"Iuliu Haţieganu" University of Medicine and Pharmacy

6 Pasteur str, Cluj-Napoca, Romania

Phone: 026-4595433

E-mail: mcrisan7@yahoo.com predilection are the knees, elbows, lower back, and scalp. The main changes of skin histology are irregular thickening of the epidermis, dilated and tortuous capillary loops in the dermis and inflammatory infiltrate represented by polymorphonuclear leucocytes and $\mathrm{T}$ lymphocytes in the upper dermis. The diagnosis is established clinically and confirmed by histology [2].

The first-line treatment for mild forms of disease consists of topical corticosteroids [3-6], with skin atrophy being the most severe potential side effect at the site of treatment $[5,6]$. The severity of the disease and the response to treatment are generally assessed using clinical scoring systems [7-9]. Clinical scores are frequently used to meet research purposes, but due to their time-consuming nature, they are not commonly applied in daily medi- 
cal practice $[1,10]$. In addition, they are subject to intraand inter-observer variation [7-9].

In this context, over the past few decades high-frequency ultrasonography (HFUS) has emerged as a valid alternative approach for morphological examination and assessment of disease progress in psoriasis [11-15]. HFUS (20 MHz and above) [16] has the advantages of being practical, widely available, non-invasive, low cost, as well as providing quantitative criteria of examination $[10,17,18]$. In plaque psoriasis, objective measurements of skin thickness have been widely used in clinical trials as indicators of effectiveness of therapy [11-14] and, to a lesser extent, as a means for early detection of steroidinduced skin atrophy $[19,20]$. More frequently, steroidinduced skin atrophy in psoriasis is assessed based on clinical examination [6]. In particular, use of low-potency corticosteroid for short periods of time is generally considered risk-free, with no development of skin atrophy [5]. However, clinically evident skin atrophy in response to six-week hydrocortisone application has been reported [21]. Detection of subclinical skin atrophy is important because it may allow prompt therapeutic intervention in view of preventing progression into irreversible damage [22].

In addition to the HFUS systems intended for skin imaging [16,17], recently developed high-frequency transducers manufactured for general-purpose ultrasonography are currently available in the market and might hold potential for dermatological application. While the efficiency of the dedicated systems has been investigated and confirmed [11-13,23], the value of the new transducers remains to be established.

The majority of general-purpose commercial systems also provide the option of tissue elasticity imaging. Sonoelastography is a relatively new technique with reported applications in skin pathology [24-26], but its dermatological potential remains insufficiently explored.

The first objective of the present study was to evaluate and compare the efficiency of $20 \mathrm{MHz}$ dedicated skin sonography and the efficiency of $40 \mathrm{MHz}$ general-purpose sonography in the assessment of psoriatic plaques under topical treatment (hydrocortisone). The second objective was to assess the efficiency of real-time sonoelastography in the early detection of steroid-induced skin atrophy in psoriatic plaques.

\section{Patients and methods}

The study was carried out upon 16 psoriatic cutaneous plaques in three consecutive patients (age: 39,50 , and 58 years) with a history of plaque psoriasis, presenting for mild clinical relapses which affected less than $10 \%$ of body surface area. The diagnosis of plaque psoriasis was established based on patient's medical history and clinical dermatological examination, which identified the presence of typical pink plaques with dry silvery-white scales. The cutaneous plaques had the following distribution: back (6), elbow (4), gluteal region (3), hip (2), and shoulder (1).

Clinical and ultrasonographic assessment was performed on each psoriatic plaque, at baseline and after six weeks of topical treatment. The treatment consisted of hydrocortisone acetate $1 \%$ ointment (Hidrocortizon ${ }^{\circledR}$; Antibiotice, Iasi, Romania) once-daily evening application and emollient once-daily morning application. None of the patients had previously been administered systemic therapy for psoriasis in the course of the disease.

Clinical assessment was documented by means of clinical photographs (Sony Cyber-Shot DSC-HX9 V, Tokyo, Japan).

Ultrasonographic assessment was performed with two equipments, each operated by a different examiner: Dermascan C (Cortex Technology, Hadsund, Denmark) with the medium-focus linear probe, operated by a dermatologist with five-year experience (MC) and Ultrasonix SonixTouch (Ultrasonix Medical Corp, Richmond, British Columbia, Canada) with the L40-8/12 linear probe, operated by a radiologist with twenty-seven-year experience in sonography, seven-year experience in elastography and two-year experience in dermatologic ultrasonography with $40 \mathrm{MHz}(\mathrm{SD})$. The operating parameters of the two equipments are shown in table I.

Table I. Operating parameters of the two ultrasound systems.

\begin{tabular}{lcc}
\hline & Dermascan & SonixTouch \\
\hline Operating frequency, MHz & 20 & 40 \\
Axial resolution, $\mu \mathrm{m}$ & 60 & 30 \\
Lateral resolution, $\mu \mathrm{m}$ & 200 & 94 \\
Field of view depth, $\mathrm{mm}$ & 15 & 8 \\
\hline
\end{tabular}

With the SonixTouch scanner each lesion underwent a B-mode and an elastographic examination. The B-mode images were interpreted according to the different echogenicity of the skin layers in the psoriatic plaque [15]. Epidermal and dermal thickness was measured on three scans of the plaque and the mean value for each layer was computed for data analysis. The epidermal thickness was measured from the upper limit of the entry echo to the lower limit of the intermediate band. The dermal thickness was measured from the lower limit of the intermediate band to the dermis-hypodermis interface [15]. 
Real-time sonoelastography was obtained by gently placing the transducer over the plaque, with a large amount of gel. Gentle vibration of the transducer over the skin was induced manually until the quality indicator scale of the machine prompted for optimal compression. For the sonoelastographic evaluation, strain ratio (SR) was used to quantify the strain difference between the dermal layer of the psoriatic plaque and the adjacent hypodermis. To compute SR, equal-sized regions of interest were placed in the dermis and in the hypodermis. In the dermis (A) and in the hypodermis (B), SR is computed as B/A. Three measurements were performed for each plaque and the mean value of SR was computed. Images of all measurements for all plaques and all examination techniques were stored digitally.

The study was approved by the local Ethics Committee and written informed consent was obtained from all patients.

\section{Statistical analysis}

Normal distribution of the data was assessed with the Kolmogorov-Smirnov test. The Student's t-test for paired samples was used to compare pre- and post-treatment pairs. The pairs were matched in order to compare epidermal and dermal thickness, respectively, as a subject of the moment when the image was taken (baseline and follow-up) and between the two ultrasound equipments. The null hypotheses were that epidermal and dermal thicknesses do not differ in response to treatment and between the two ultrasound equipments. For SR, the significance of the difference between baseline and follow-up values was assessed, with the null hypothesis that there was no difference before and after treatment. In all tests, a confidence level of $p<.05$ was considered significant. The statistical tests were performed using the R program, version 3.0.2.

\section{Results}

On clinical exam, all plaques showed improvement of the clinical presentation following treatment, with reduced erythema, induration, and scaling (fig 1).

\section{$B$-mode ultrasonographic evaluation of treatment response}

On the ultrasound images acquired with both sonographic equipments psoriatic plaques presented as three-layered lesions. The superficial layer was highly echogenic, the intermediate layer appeared hypoechoic to anechoic and the profound layer was of intermediate echogenicity. This pattern was observed in all lesions at baseline. At follow-up the intermediate layer was visibly reduced or even undetectable (fig 2).

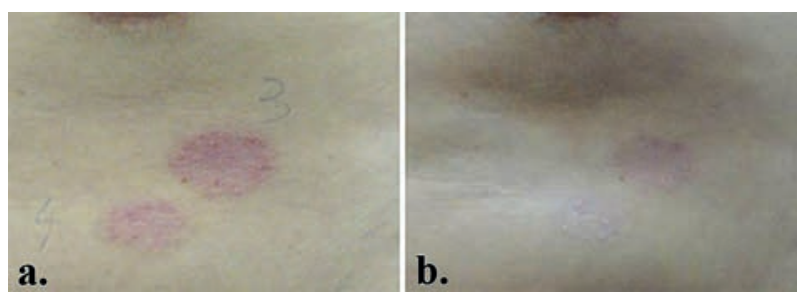

Fig 1. Clinical appearance of psoriatic plaques in a 50 year-old patient: a. at presentation; b. at follow-up.
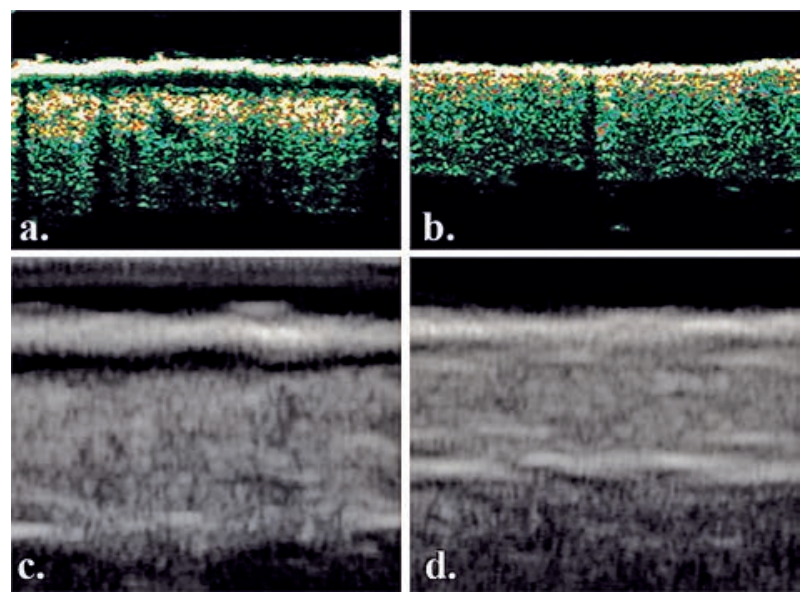

Fig 2. B scale appearance of a psoriatic plaque located on the back in a 50 year-old man: a. and b. Dermascan $\mathrm{C}$ sonograms $(20 \mathrm{MHz})$ at baseline and at follow-up, respectively; c. and d. SonixTouch sonograms (40 MHz) at baseline and at follow-up, respectively.

The paired-samples t-test for the $20 \mathrm{MHz}$ measurements found a significant difference in epidermal thickness scores in response to treatment (baseline: $0.21 \pm$ 0.059; follow-up: $0.15 \pm 0.04 ; \mathrm{p}=.002$ ). Results were similar for the $40 \mathrm{MHz}$ measurements of epidermal thickness (baseline: $0.71 \pm 0.18$; follow-up: $0.60 \pm 0.13$, $\mathrm{p}=.032$ ). Follow-up $20 \mathrm{MHz}$ evaluation suggested an increase in dermal thickness (baseline: $1.51 \pm 0.45$; followup: $1.61 \pm 0.25 ; \mathrm{p}=.35$ ), shown to be insignificant. The corresponding $40 \mathrm{MHz}$ values did not show a significant reduction in dermal thickness (baseline: $2.18 \pm 0.67$; follow-up: $2.05 \pm 0.50 ; \mathrm{p}=.33$ )

Comparison of the ultrasound equipments $(20 \mathrm{MHz}$ - $40 \mathrm{MHz}$ variation)

The mean values of epidermal and dermal thicknesses determined at the frequency of $40 \mathrm{MHz}$ were higher than the $20 \mathrm{MHz}$ counterparts in all the patients. Pairedsamples t-test to test for the significance of the variation showed highly significant differences in epidermal thickness at baseline $(20 \mathrm{MHz}: 0.21 \pm 0.059$; $40 \mathrm{MHz}$ : 0.70 $\pm 0.18 ; \mathrm{p}<.001)$ and at follow-up (20 MHz: $0.15 \pm 0.04$; 


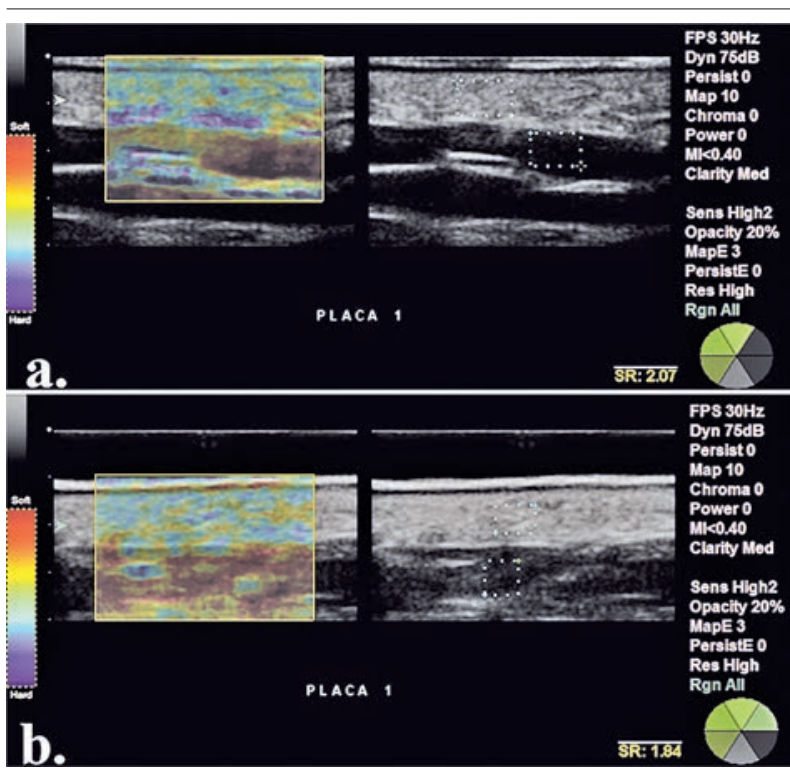

Fig 3. Sonoelastographic appearance of a psoriatic plaque located on the back in a 39 year-old woman: a. Baseline sonoelastogram; b. Follow-up image.

$40 \mathrm{MHz}: 0.59 \pm 0.13 ; \mathrm{p}<.001)$. The differences in dermal thickness were significant at baseline (20 MHz: $1.51 \pm$ 0.45 ; $40 \mathrm{MHz}: 2.24 \pm 0.66 ; \mathrm{p}=.003)$ and at follow-up (20 MHz: $1.61 \pm 0.25 ; 40 \mathrm{MHz}: 2.11 \pm 0.47 ; \mathrm{p}=.001$ ).

\section{Sonoelastographic evaluation of steroid-induced skin atrophy}

The hypodermis exhibited a constant dark red signal (softest). Dermal elastographic signal showed highly variable patterns. Both initial and final elastograms displayed various combinations of mixed blue and green bands of color, a preponderance of red signals, scattered areas of pale blue and red spotted signals (fig 3). Treatment was not found to influence dermal elasticity, SR variation being statistically insignificant (baseline: $1.61 \pm 0.75$; follow-up: $1.60 \pm 0.60 ; \mathrm{p}=0.96$ ).

\section{Discussions}

Ultrasonography in dermatology employs two frequency ranges: 7.5-15 $\mathrm{MHz}$ for regional lymph nodes and cutaneous tumors $[16,17,27]$ and $20-100 \mathrm{MHz}$ for skin morphology examination [11-14,16,23,28]. Highresolution dermatologic ultrasonography is commonly performed at $20 \mathrm{MHz}$ [16-18].

In normal glabrous skin, $20-40 \mathrm{MHz}$ ultrasonography shows the dermal layer directly below the entrance echo $[18,28]$, whereas $100 \mathrm{MHz}$ ultrasonography allows for the visualization of an additional intermediate anechoic band, located between the entrance echo and the reticu- lar dermis and representing the viable epidermis plus the papillary dermis [28].

In psoriatic plaques, the histological modifications generate corresponding changes in sonographic morphology: hyperkeratosis and acanthosis determine an increase in epidermal thickness; inflammation and vascular abnormalities determine an increase in dermal thickness [29]. Due to hyperkeratosis and superficial scaling the stratum corneum becomes thicker and hyperechoic, which determines an increase in the thickness and echogenicity of the entrance echo [13,28]. Epidermal acanthosis and inflammation of the papillary dermis determine an increase in the thickness of the intermediate band, which allows for its representation on 20-40 MHz sonograms [13,14,28].

Psoriatic plaques have been investigated at various frequencies within the 20-100 MHz range [11-14,28,30]. High frequencies directly correlate with a better axial resolution, which is important to discriminate between individual skin layers [16,18]. However, even in the context of the high resolution provided at the frequency of $100 \mathrm{MHz}$, the dermo-epidermal junction cannot be visualized [28]. In addition, with the increase in frequency, the penetration depth of the ultrasonic wave decreases $(1.5 \mathrm{~mm}$ for 100 $\mathrm{MHz})[16,18]$. With whole skin thickness (epidermis plus dermis) in untreated psoriatic plaques reaching dimensions of up to $3.5 \mathrm{~mm}$ [20], lower frequency ranges may be more adequate to investigate psoriatic skin lesions.

Numerous studies indicate that the thickness of the intermediate band is the most important sonographic parameter which correlates with severity and treatment response in psoriatic plaques [12-14,30]. However, the intermediate band is not characteristic for psoriatic plaques [18,31]. In psoriasis, Hoffmann et al found a decrease in the thickness of the intermediate band following treatment by $99.92 \%$ [13].

In the present study, epidermal thickness measurements included the entrance echo and the intermediate band. This implied an overestimation of the real epidermal thickness due to the fact that the entrance echo and the intermediate band also contain the echoes from the water-skin interface and the papillary dermis, respectively. In response to therapy, the decrease in epidermal thickness measured as described above, is an expression of two components. The first component is the normalization of the epidermal structure with reduced scaling at clinical assessment. Sonographically, this involves a reduction in the thicknesses of the entrance echo and of the intermediate band due to diminished hyperkeratosis and acanthosis, respectively. The second component refers to the amelioration of dermal pathological changes with reduced erythema at clinical assessment and a further decrease in the thickness of the intermediate band due to receding inflammation in the papillary dermis $[13,28]$. 
In our study, dermal thickness measurements refer specifically to the reticular dermis. Contrary to the results of this study, Hoffmann et al [13] found a specific decrease in the thickness of the reticular dermis in response to treatment by $45.2 \%$. Vaillant et al [12] found a mean increase in dermal thickness in psoriatic lesions by $60 \%$ when compared to normal skin and reported a decrease in dermal thickness in response to treatment. However, the dermal measurements in their study included the intermediate band. Therefore, the decrease in dermal thickness in response to treatment cannot specifically be attributed to the reticular dermis. In accordance with the results of this study, El-Zawahry et al [30] reported that dermal thickness showed no significant difference in psoriatic skin vs. normal skin and no correlation with the clinical severity of the disease as assessed with the PASI score. This is in agreement with the pathological changes which develop in psoriasis. Creamer et al [32] demonstrated that the most prominent pathogenetic feature of psoriasis, i.e. the dermal microvascular proliferation [33], was exclusively located in the papillary dermis and thus it did not involve the reticular dermis.

The difference between the measurements acquired with the two ultrasound equipments can be attributed to the potential inter-observer variability and the different image construction techniques used by the two systems.

Another aim of this study was to investigate the potential of sonoelastography for the early detection of steroid-induced skin atrophy in psoriatic plaques. Ultrasound-based elastography is the most widely used technique for soft tissue elasticity mapping. Tissue mechanical properties are imaged in response to external deformation. The skin, being susceptible to external deformation, might prove to be an adequate target for sonoelastographic investigation [34].

Steroid-induced skin atrophy involves both the epidermis and the dermis [35]. In the natural course of steroid atrophy, the first event is represented by a reduction in epidermal thickness [5], which is potentially reversible with early treatment readjustment [36]. Extended steroid use leads to additional dermal deterioration and striae formation which, unlike epidermal atrophy, tend to be persistent or irreversible [22]. This aspect, along with a potential tendency to clinically underestimate steroidinduced skin atrophy in psoriatic plaques [6], emphasizes the need for an early objective determination of dermal atrophic changes prior to their clinical expression.

In the present study, the use of sonoelastography to meet the aforementioned need relies on the observation that steroid-induced dermal atrophy is associated with loss of dermal elasticity [37-39]. Josse et al [38] found a good correlation between skin mechanical properties and histological assessment in atrophic skin.
High-frequency ultrasonography has been used to determine dermal thickness variation in response to topical steroid treatment in psoriatic plaques $[19,20]$. However, it has been reported that dermal mechanical properties vary to a greater extent compared to dermal thickness determinations (35\% and 10\%, respectively) [38]. Therefore, sonoelastography was used in this study in order to look further into the dermal information acquired with B-mode sonography.

Strain ratio values higher than 1 signified increased dermal stiffness relative to the hypodermis. This is not specific to psoriasis, because in normal skin the dermis typically exhibits higher stiffness compared to the hypodermis [40]. The insignificant variation of SR values following treatment may allow to hypothesize on the absence of dermal atrophy in this group of patients, which would be in accordance with the expected course of events with low-potency steroid use for short periods of time [5].

The limitations of the study include the small number of patients enrolled and the lack of a control group. Being a work-in-progress study and involving examiners with a wide gamut of expertise in different technologies and equipments, an intra- and inter-observer variability test, especially for sonoelastography, could have further refined the critical analysis of the observations.

\section{Conclusions}

Both skin dedicated sonography using $20 \mathrm{MHz}$ frequency and general-purpose sonograpghy using $40 \mathrm{MHz}$ frequency allowed for unequivocal imaging and morphological interpretation of psoriatic plaques. Although the two techniques provided significantly different measurements of sonographic parameters, each technique was found to be efficient at quantifying treatment response in psoriatic plaques.

To the best of our knowledge, there are no previous studies on plaque psoriasis which compare skin dedicated sonography with general-purpose sonography or which apply sonoelastography in the assessment of steroid-induced skin atrophy.

Real-time sonoelastography of psoriatic lesions with SR variation analysis did not show a significant change of dermal elasticity following short-term application of low-potency topical steroid (hydrocortisone). The value of sonoelastography in the early subclinical detection of steroid-induced skin atrophy is a concept worthy of further investigation, possibly in the context of long-term steroid use or high-potency steroid application.

Conflict of interest: none 


\section{References}

1. Menter A, Gottlieb A, Feldman SR, et al. Guidelines of care for the management of psoriasis and psoriatic arthritis: Section 1. Overview of psoriasis and guidelines of care for the treatment of psoriasis with biologics. J Am Acad Dermatol 2008; 58: 826-850.

2. Cohen SN, Baron SE, Archer CB; British Association of Dermatologists and Royal College of General Practitioners. Guidance on the diagnosis and clinical management of psoriasis. Clin Exp Dermatol 2012; 37 Suppl 1: 13-18.

3. Lebwohl M. Psoriasis. Lance. 2003; 361: 1197-1204.

4. Menter A, Korman NJ, Elmets CA, et al; American Academy of Dermatology. Guidelines of care for the management of psoriasis and psoriatic arthritis. Section 3. Guidelines of care for the management and treatment of psoriasis with topical therapies. J Am Acad Dermatol 2009; 60: 643-659.

5. Del Rosso J, Friedlander SF. Corticosteroids: options in the era of steroid-sparing therapy. J Am Acad Dermatol 2005; 53 (1 Suppl 1): S50-S58.

6. Castela E, Archier E, Devaux S, et al. Topical corticosteroids in plaque psoriasis: a systematic review of risk of adrenal axis suppression and skin atrophy. J Eur Acad Dermatol Venereol 2012; 26 Suppl 3: 47-51.

7. Ashcroft DM, Wan Po AL, Williams HC, Griffiths CE. Clinical measures of disease severity and outcome in psoriasis: a critical appraisal of their quality. Br J Dermatol 1999;141:185-191.

8. Marks R, Barton SP, Shuttleworth D, Finlay AY. Assessment of disease progress in psoriasis. Arch Dermatol 1989; 125: 235-240.

9. Puzenat E, Bronsard V, Prey S, et al. What are the best outcome measures for assessing plaque psoriasis severity? A systematic review of the literature. J Eur Acad Dermatol Venereol 2010;24 Suppl 2:10-16.

10. Wortsman X. Common applications of dermatologic sonography. J Ultrasound Med 2012; 31: 97-111.

11. Di Nardo A, Seidenari S, Giannetti A. B-scanning evaluation with image analysis of psoriatic skin. Exp Dermatol 1992; 1: 121-125.

12. Vaillant L, Berson M, Machet L, Callens A, Pourcelot L, Lorette G. Ultrasound imaging of psoriatic skin: a noninvasive technique to evaluate treatment of psoriasis. Int $\mathrm{J}$ Dermatol 1994; 33: 786-790.

13. Hoffmann K, Dirschka T, Schwarze H, et al. $20 \mathrm{MHz}$ sonography, colorimetry and image analysis in the evaluation of psoriasis vulgaris. J Dermatol Sci 1995; 9: 103-110.

14. Gupta AK, Turnbull DH, Harasiewicz KA, et al. The use of high-frequency ultrasound as a method of assessing the severity of a plaque of psoriasis. Arch Dermatol 1996; 132: 658-662.

15. Gutierrez M, Wortsman X, Filippucci E, De Angelis R, Filosa G, Grassi W. High-frequency sonography in the evaluation of psoriasis: nail and skin involvement. J Ultrasound Med 2009; 28: 1569-1574.

16. Jasaitiene D, Valiukeviciene S, Linkeviciute G, Raisutis R, Jasiuniene E, Kazys R. Principles of high-frequency ultra- sonography for investigation of skin pathology. J Eur Acad Dermatol Venereol 2011; 25: 375-382.

17. Schmid-Wendtner MH, Burgdorf W. Ultrasound scanning in dermatology. Arch Dermatol 2005; 141: 217-224.

18. Jemec GB, Gniadecka M, Ulrich J. Ultrasound in dermatology. Part I. High frequency ultrasound. Eur J Dermatol 2000; 10: 492-497.

19. Andres P, Poncet M, Farzaneh S, Soto P. Short-term safety assessment of clobetasol propionate $0.05 \%$ shampoo: hypothalamic-pituitary-adrenal axis suppression, atrophogenicity, and ocular safety in subjects with scalp psoriasis. J Drugs Dermatol 2006; 5: 328-332.

20. Lacarrubba F, Nardone B, Musumeci ML, Micali G. U1trasound evaluation of clobetasol propionate $0.05 \%$ foam application in psoriatic and healthy skin: a pilot study. Dermatol Ther 2009; 22 Suppl 1: S19-S21.

21. Katz HI, Prawer SE, Watson MJ, Scull TA, Peets EA. Mometasone furoate ointment $0.1 \%$ vs. hydrocortisone ointment $1.0 \%$ in psoriasis. Atrophogenic potential. Int J Dermatol 1989; 28: 342-344.

22. Horn EJ, Domm S, Katz HI, Lebwohl M, Mrowietz U, Kragballe K; International Psoriasis Council. Topical corticosteroids in psoriasis: strategies for improving safety. J Eur Acad Dermatol Venereol 2010; 24: 119-124.

23. Turnbull DH, Starkoski BG, Harasiewicz KA, et al. A 40$100 \mathrm{MHz}$ B-scan ultrasound backscatter microscope for skin imaging. Ultrasound Med Biol 1995; 21: 79-88.

24. Gaspari R, Blehar D, Mendoza M, Montoya A, Moon C, Polan D. Use of ultrasound elastography for skin and subcutaneous abscesses. J Ultrasound Med 2009; 28: 855-860.

25. Iagnocco A, Kaloudi O, Perella C, et al. Ultrasound elastography assessment of skin involvement in systemic sclerosis: lights and shadows. J Rheumatol 2010; 37: 1688-1691.

26. Deprez JF, Brusseau E, Fromageau J, Cloutier G, Basset O. On the potential of ultrasound elastography for pressure ulcer early detection. Med Phys 2011; 38: 1943-1950.

27. Dudea SM, Lenghel M, Botar-Jid C, Vasilescu D, Duma M. Ultrasonography of superficial lymph nodes: benign vs. malignant. Med Ultrason 2012; 14: 294-306.

28. El Gammal S, El Gammal C, Kaspar K, et al. Sonography of the skin at $100 \mathrm{MHz}$ enables in vivo visualization of stratum corneum and viable epidermis in palmar skin and psoriatic plaques. J Invest Dermatol 1999; 113: 821-829.

29. Schön MP, Boehncke WH. Psoriasis. N Engl J Med 2005; 352: 1899-1912.

30. El-Zawahry MB, Abdel El-Hameed El-Cheweikh HM, Abd-El-Rahman Ramadan S, Ahmed Bassiouny D, Mohamed Fawzy M. Ultrasound biomicroscopy in the diagnosis of skin diseases. Eur J Dermatol 2007; 17: 469475.

31. Crisan D, Lupsor M, Boca A, Crisan M, Badea R. Ultrasonographic assessment of skin structure according to age. Indian J Dermatol Venereol Leprol 2012; 78: 519.

32. Creamer D, Allen MH, Sousa A, Poston R, Barker JN. Localization of endothelial proliferation and microvascular expansion in active plaque psoriasis. Br J Dermatol 1997; 136: 859-865. 
33. Heidenreich R, Röcken M, Ghoreschi K. Angiogenesis drives psoriasis pathogenesis. Int J Exp Pathol 2009; 90: 232-248.

34. Wells PN, Liang HD. Medical ultrasound: imaging of soft tissue strain and elasticity. J R Soc Interface 2011; 8: 15211549 .

35. Cossmann M, Welzel J. Evaluation of the atrophogenic potential of different glucocorticoids using optical coherence tomography, 20-MHz ultrasound and profilometry; a double-blind, placebo-controlled trial. Br J Dermatol 2006; 155: 700-706.

36. Katz HI, Prawer SE, Mooney JJ, Samson CR. Preatrophy: covert sign of thinned skin. J Am Acad Dermatol 1989; 20: 731-735.
37. Hengge UR, Ruzicka T, Schwartz RA, Cork MJ. Adverse effects of topical glucocorticosteroids. J Am Acad Dermatol 2006; 54: 1-15.

38. Josse G, Rouvrais C, Mas A, et al. A multitechnique evaluation of topical corticosteroid treatment. Skin Res Technol 2009; 15: 35-39.

39. Lehmann P, Zheng P, Lavker RM, Kligman AM. Corticosteroid atrophy in human skin. A study by light, scanning, and transmission electron microscopy. J Invest Dermatol 1983; 81: 169-176.

40. Vogt M, Ermert H. Development and evaluation of a highfrequency ultrasound-based system for in vivo strain imaging of the skin. IEEE Trans Ultrason Ferroelectr Freq Control 2005; 52: 375-385. 